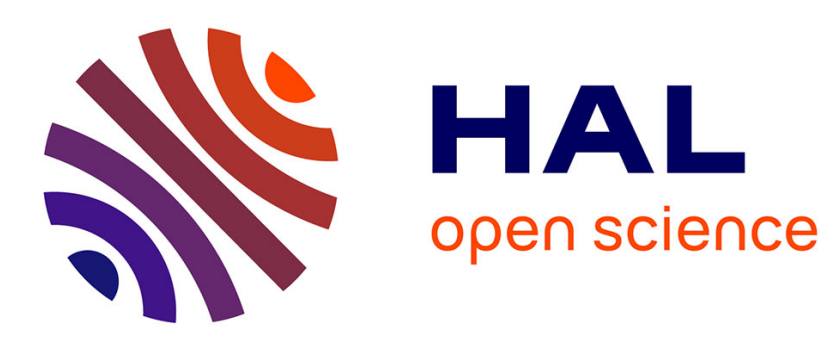

\title{
Discours de soi dans le champ de la santé publique. Engagement et expérimentation vaccinale.
}

\author{
Pascale Delormas
}

\section{To cite this version:}

Pascale Delormas. Discours de soi dans le champ de la santé publique. Engagement et expérimentation vaccinale. . 2017. hal-01654364

\section{HAL Id: hal-01654364 \\ https://hal.science/hal-01654364}

Preprint submitted on 3 Dec 2017

HAL is a multi-disciplinary open access archive for the deposit and dissemination of scientific research documents, whether they are published or not. The documents may come from teaching and research institutions in France or abroad, or from public or private research centers.
L'archive ouverte pluridisciplinaire HAL, est destinée au dépôt et à la diffusion de documents scientifiques de niveau recherche, publiés ou non, émanant des établissements d'enseignement et de recherche français ou étrangers, des laboratoires publics ou privés. 


\section{Discours de soi dans le champ de la santé publique Engagement et expérimentation vaccinale}

Depuis quelques années nous interrogeons les usages discursifs de légitimation des individus en fonction des communautés dans lesquels ils évoluent, en d'autres termes, à l'articulation des identités discursives et des lieux sociaux. Si «l'opération de légitimation» est contingente, si elle "prend des formes différentes selon les situations d'énonciation, les formes de communication, les problèmes débattus et le type de lien qui existe entre les locuteurs » (Ebel 1981: 26), les enjeux en fonction desquels les locuteurs agissent et interagissent sont en général peu explicites alors que des postures spécifíques aux communautés discursives sont attendues. Les discours de soi comme tous les types de discours, loin d'être l'expression de la seule individualité, sont eux aussi soumis à des rites et des modes de socialisation spécifiques, à des "régimes » différents (singularisation, acculturation, coopération, etc.), dont la connaissance permet aux individus de trouver leur place, de s'imposer et de se faire valoir pour exister.

Alors qu'un numéro de la revue Adarr publié en 2015 mobilise la notion d'ethos collectif ${ }^{1}$ pour analyser la construction des identités publiques dans l'action collective et la présentation de soi des groupes militants dans les discours de l'action collective, il me semble que l'ethos de «l'engagement individuel » à un projet collectif entendu comme coconstruction n'a pas encore bénéficié d'un traitement spécifique. La participation des acteurs non experts à la recherche médicale est un exemple de ce type d'engagement. L'analyse montre dans quelle mesure les discours de présentation de soi de ces individus relèvent du régime de participation et l'on peut sans doute considérer qu'ils prennent part à l'exercice de ce que Foucault nomme le biopouvoir.

\section{Corpus et cadre méthodologique}

Le corpus des trente-quatre échanges verbaux examiné est ancré dans le terrain de la recherche médicale et de la communication en matière de santé publique. Il s'agissait, dans le cadre d'une commande d'un centre hospitalier régional et en vue d'obtenir des profils susceptibles de susciter des vocations, d'améliorer la politique de recrutement de volontaires dans des essais vaccinaux contre le VIH à travers l'expression de leur motivation. Les entretiens semi-directifs ont été menés par une anthropologue de la santé ${ }^{2}$. Ces entretiens ont été retranscrits à partir des interviews sans qu'il soit tenu compte de toutes les marques de l'énonciation qu'un analyste du discours souhaiterait avoir à sa disposition ; cependant l'approche sémantico-discursive adoptée dans notre analyse qualitative permet de mener l'analyse de l'ethos discursif et de dégager la spécificité de ces «discours de soi participatifs $\pi$.

Rappelons la définition de la sémantique discursive (versus sémantique linguistique) que donne Charaudeau (2005) :

«La sémantique de discours présuppose l'existence d'une sémantique de langue (la première ne peut exister sans la seconde), il faut accepter que la seule

\footnotetext{
${ }^{1}$ En Analyse de discours on parle d'ethos discursif (même si l'on ne néglige pas l'extraverbal) c'est-à-dire que l'on prend en considération les seuls énoncés, envisagés dans leur contexte, pour définir l'image de soi - ou encore l'éthos qui en émerge. Il ne s'agit pas de l'ethos aristotélicien qui prend en compte la personne physique de l'orateur, sa façon d'être.

${ }^{2}$ Les entretiens ont été menés par Mathilde Couderc, post-doctorante rattachée au Céditec dans le cadre de l'intégration de ce laboratoire dans le Labex VRI, et du partenariat avec le Centre Hospitalier Intercommunal de Créteil, labellisé "Centre de recherche clinique ». - Prévention du sida et vaccin : débats publics et médiatiques, pratiques informationnelles et engagement dans un essai vaccinal.
} 
sémantique de langue ne peut prétendre à rendre compte du sens des actes de langage produits en situation de communication réelle: elle a besoin d'être complétée par une sémantique de discours.

Mais dans un cas comme dans l'autre, il s'agit de «traquer» le sens. Dans un cas, ce sera à travers un calcul d'ordre probabiliste, dans l'autre à travers un calcul inférentiel non nécessairement prévisible (ni prédictible), sur un même terreau sémantique de virtualités, et toujours dans un mouvement de va-et-vient entre le général et le particulier. »

La notion d'ethos, considérée comme un articulateur majeur en analyse de discours, permet de travailler du point de vue de linguistique comme du point de vue des pratiques sociales ; en cela elle donne accès à la dimension idéologique du discours. L'ethos discursif des propos des volontaires engagés dans l'expérimentation du vaccin contre le VIH est analysé selon une tripartition qui en tient compte de la dimension expérientielle, de la dimension catégorielle et de la dimension idéologique de l'ethos que Maingueneau (2014: 32-33) définit ainsi :

[...] je propose à présent d'attribuer à l'éthos trois dimensions (catégorielle, expérientielle et idéologique) qui sont plus ou moins saillantes selon les textes considérés : 1) la dimension "catégorielle» recouvre des choses très diverses. Il peut s'agir de rôles discursifs ou de statuts extradiscursifs. Les rôles discursifs sont ceux qui sont liés à l'activité de parole [...]. Les statuts extradiscursifs peuvent être de natures très variées $[\ldots]$; 2) la dimension «expérientielle » de l'éthos recouvre les caractérisations socio-psychologiques stéréotypiques, associées aux notions d'incorporation et de monde éthique [...] 3) la dimension « idéologique » renvoie à des positionnements dans un champ [...]. Ces trois dimensions interagissent fortement. Le paysan (catégoriel) a des affinités stéréotypiques avec le bon sens (expérientiel) et le conservatisme (idéologique) [...].

\section{Dimension expérientielle de l'ethos}

Parmi les indices discursifs que l'on peut relever dans les entretiens analysées, le trait stéréotypique le plus prégnant est, comme on peut s'y attendre, celui de l'altruisme et les trois traits psychologiques susceptibles d'être associés à l'expression de l'engagement dans de tels essais vaccinaux sont celui de la responsabilité, de la générosité et de l'enthousiasme.

\section{La responsabilité, l'exemplarité de son action}

«Moi j'aime pás trop voir les choses comme ça, et je pense que surtout en santé publique, en promotion de la santé, il faut que les gens se saisissent de leur santé et se saisissent des enjeux de santé. » (C'est moi qui souligne, comme dans les passages qui suivront)

« Ça s'inscrit dans une démarche globale, c'est ma vraie personnalité qui est comme ça, et je pense que si tout le monde... Enfin, pas tout le monde, parce que c'est pas faisable, mais si beaucoup plus de gens donnaient leur sang par exemple, ce qui est quelque chose de très simple, ça ferait énormément de progrès. »

\section{La générosité : le don de son corps à la science}

«Enfin, c'est pas banal non plus, on prête son corps quelque part. Moi je suis déjà un donneur de sang régulier aussi. »

« et je le fais pas pour moi - enfin, on le fait toujours un peu pour soi, mais je le fais aussi surtout pour d'autres gens quoi. Et surtout pour cette maladie là. Je 
pense que les cancers, tout ça, ça n'aurait pas du tout été la même chose. C'était vraiment le VIH. [....] et de m'impliquer personnellement quoi. Intimement on va dire. Parce que c'est le corps, c'est tout ça quoi. Parce que là, je vais le faire d'un point de vue professionnel, donc ça c'est une chose, mais après il y a l'intime aussi quoi. "

\section{L'enthousiasme}

Au sens fort du terme, l'enthousiasme signifie une dévotion complète à un idéal, une cause, qui se traduit par de la joie et de l'excitation. L'engagement actif du corps est vécu souvent par les volontaires interrogés comme élément de complétude, dans une véritable communion avec l'humanité :

« J'avais ça très à cœur et donc j'ai dû faire un peu le deuil ${ }^{3}$, que jamais de mon vivant je donnerai aux personnes qui en avaient besoin. [...] je donne tout moi, la totale!»

« Je suis quelqu'un qui aime bien donner de ma personne, donc du coup si je peux aider, j'aide ! »

\section{Dimension catégorielle de l'ethos}

L'image du locuteur, c'est-à-dire l'éthos discursif, relève d'une co-construction et, cela, sur deux plans, celui de l'ethos préalable (ou «ethos projeté » a priori sur les volontaires engagés dans l'expérimentation) et celui de l'ethos qui se dégage de leurs dires, en relation directe avec les relances de l'enquêteur. La représentation que nous nous faisons des volontaires investis dans l'action publique contre le VIH pourrait renvoyer à un ethos catégoriel selon lequel ils seraient de gauche, jeunes, de profession médicale ou paramédicale, homosexuels, etc... or, en fait, l'hétérogénéité de statut des locuteurs interviewés investis dans l'expérimentation montre qu'ils ne constituent pas une catégorie bien définie.

Outre le fait qu'ils ne sont pas recrutés sur des critères sociologiques, les volontaires sont mobilisés selon une configuration spatiale et temporelle qui ne les constituent pas davantage comme groupe (il n'est pas question dans cette action de recrutement de réunir les personnes engagées dans l'expérimentation). Les candidats aux essais vaccinaux sont interpellés individuellement par la campagne (via des journaux gratuits, des affichages, etc...), ils mènent seuls une investigation documentaire et ils consultent seuls les professionnels de la santé investis dans le projet.

Ils constituent cependant ún groupe pour les médecins qui cherchent à réunir des individus pour un panel d'expérimentation aussi diversifié que possible et pour le chercheur qui collecte des données de terrain pour construire son corpus. A un niveau discursif, ces individus peuvent donc être définis par leur activité de parole : ils ont accepté de participer à une interaction verbale dans un entretien semi-directif, à l'occasion duquel ils font un retour réflexif sur lés raisons de leur engagement dans des essais vaccinaux.

\subsection{Individuel versus collectif : des notions à articuler en termes de degrés d'appartenance} On constate que la dimension catégorielle de l'ethos est problématisée par les volontaires eux-mêmes. Cela conduit à interroger le type de relation des individus au collectif comme le fait par exemple Pinto (2009) dans son ouvrage Le collectif et l'individuel. Considérations durkheimiennes :

«Les notions de groupe et de représentation collective sont indispensables pour rendre compte de la transcendance qui caractérise certains êtres dans le monde social... Il y a dans l'individu des choses qui ne viennent pas de lui et qui ont une

\footnotetext{
${ }^{3}$ Il s'agit d'une volontaire qui n'a finalement pas été recrutée dans cette campagne d'essais vaccinaux.
} 
forme d'objectivité spécifique. Mais si le groupe se distingue de ses membres singuliers, il n'existe que par leur action qui les fait exister en tant que tels. Il y a donc des degrés d'appartenance et de cohésion, et l'on peut parfaitement étudier empiriquement les modes d'être du collectif, leurs différences de constitution et d'intensité. » (Pinto 2009 : 146)

Même s'ils se savent nombreux à coopérer à une action sanitaire, les volontaires ne se conçoivent pas comme membres d'un groupe : ils parlent de la façon dont ils ont été interpellés individuellement ("interpellation » est pris ici dans son sens courant mais aussi dans le sens de « convocation » que lui donne Althusser) et/ou alors ils regrettent l'absence de collectif.

Mais la représentation du collectif est bien présente dès lors que la possibilité qu'elle existe est pointée. Ainsi, les locuteurs évoquent-ils des catégories dont ils feraient partie pour expliquer les raisons de leur sensibilité ou de leur intérêt pour la recherche sur le VIH, comme le fait par exemple Brian, étudiant dans le secteur de la biologie :

«J'étais pas vraiment un «monsieur-tout-le-monde» quand j'ai vu... Parce qu'étant déjà en biologie et étant déjà naturellement intéressé par ces enjeux-là, je suis déjà, mon cerveau a déjà été conditionné à... Donc c'est pour ça que j'étais limite la personne, le bon poisson à ferrer tout de suite quói. »"

Si la démarche est individuelle, le désir de regroupement est manifeste. Si les volontaires ne constituent pas une communauté effective parce qu'ils ne se rencontrent pas, ils le déplorent et se réclament d'une communauté imaginaire:

«Ça ne me faisait pas peur, je trouvais ça mếme intéressant. Je me disais, ce statut sérologique, j'ai en commun quelque chose avec des gens qui vivent ça vraiment. J'aurais bien aimé rencontrer d'autres gens pour en discuter. [.....] Moi ça m'intéresserait énormément de rencontrer [...]. Et justement, d'échanger sur les parcours de chacun, qu'est-ce qui les a amené à faire ça... Mais ne seraitce que d'un point de vue personnel je trouverai ça extrêmement enrichissant. »

Parfois une hésitation se manifeste quant à la bonne distance à préserver entre l'intime et collectif. Ainsi :

«Je trouve que d'ailleurs c'est quelque chose qui manque. Si moi j'ai une suggestion à apporter c'est que dans ce genre de chose c'est vraiment bien de créer un réseau de volontaires. Que les gens se rencontrent un peu. C'est quand même assez intéressant de pouvoir partager avec des gens qui sont aussi intéressés par ce genre de démarche. [...] Je sais pas sous quelle forme il pourrait y avoir cette communauté un petit peu, sans forcément que ce soit quelque chose de trop lourd à alimenter. [...] ça peut être sympa de se sentir dans une communauté de gens qui ont les mêmes envies à cœur. »

\subsection{Référence à une instance transcendante}

L'absence d'un marquage énonciatif du collectif - « nous » ou « on » n'apparaissent pas ne contrevient pas la référence très fréquente à un surdestinataire, une entité transcendante, comme «l'humanité », qui confère une mission à ces volontaires. À ce stade, selon un paradoxe qui n'est qu'apparent, l'intensité de l'appartenance est d'autant plus forte que cette instance est fantasmée.

«Je pense qu'il y avait vraiment cette idée d'être utile. Être utile pour quelque chose qui me semble extrêmement important et crucial. Globalement, j'essaie 
toujours d'avoir une pensée un peu large sur les choses, et c'est pour ça aussi que je me suis intéressée à la santé publique, à la santé par la santé publique, d'un point de vue populationnel, d'un point de vue mondial. »

ou « la science », « la médecine » - aux efforts de laquelle ils participent, dans la foi dans le progrès :

«Ben c'est pour faire avancer la science! Non, c'est vrai, c'est important. Et puis même, au-delà de faire un don à l'institut Pasteur, un don financier, ou quoi que ce soit, c'est aussi bien de... »

« De se dire qu'on participe à quelque chose qui... Enfin, à de la recherche, et que dans tous les cas, que ce soit positif ou négatif, ça fera avancer. On en connaitra un peu plus sur la maladie et la façon de la guérir aussi. »"

«C'était sympa de se dire qu'il y avait des choses qui étaient faites pour améliorer la situation, donc c'était normal de vouloir participer à ça. [...] l'envie de contribuer à la recherche médicale de manière générale. De manière générale, on avait un vrai impact en tant que volontaire. Mais je pense qu'il n'y a pas vraiment d'autre façon pour avoir un vrai impact pour ça. »

La médiation pour un accès à cette transcendance s'incarne dans l'équipe médicale. L'enrôlement dans l'expérimentation est en effet parfois vécu comme une élection, une intronisation dans le milieu des experts.

«J'aimerais bien participer à tout ce milieu de la recherche, j'aimerais bien devenir chercheur, par rapport aux virus tout ça, c'est vraiment un truc qui m'intéresse. Et du coup, en attendant d'avoir les outils intellectuels pour pouvoir aller dans ce sens-là, ben j'essaie de me rendre utile comme je peux. Je mets la pierre à l'édifice un petit peu, à ma façon quoi, enfin à mon niveau, disons. Mais ouais... Le truc qui pourrait êtré sympa c'est que ce monsieur, ce médecin, que je puisse avoir un petit stage ce serait cool. »

« Je fais peut-être un peu de la psychologie de comptoir, mais peut-être que je recherche aussi un substitut au fait que j'ai pas fait d'études médicales et que oui, je suis pas une technicienne dans le domaine, mais en revanche j'ai une culture scientifíque développée. [...] Et puis bon, après je défend beaucoup le fait que même si on est non spécialiste de quelque chose on a le droit de... Bon, les médecins ils savent que souvent c'est un petit peu... Ils ont le statut, ils ont le savoir, ils sont experts dans leurs domaines, et puis en face on a des profanes qui, souvent, doivent un peu accueillir la bonne parole ... ça me semblait intéressant de me saisir d'un enjeu de santé publique. [...] Je me suis dit voilà, faire partie du commun des mortels et pouvoir aider des médecins qui travaillent là-dessus. »

« Il y a pas énormément de monde qui participe à l'essai, donc du coup ben ça fait plaisir de se dire qu'on fait partie de ces personnes-là. »

De façon plus diffuse, la posture critique qui consiste à envisager l'amélioration de la communication à propos des essais vaccinaux ou la manifestation de l'intention d'en faire connaître l'existence à son entourage peuvent être interprétées comme des signes de l'attraction qu'exerce le pouvoir/savoir médical et de la possibilité de développer une expertise du fait de leur expérience. 
«Ce serait peut-être bon pour eux de travailler leur image auprès de la population pour vraiment être identifiable. Parce qu'ils jouent quand même un rôle très important pour notre société, trouver des vaccins des trucs comme ça, c'est quand même important quoi. »

« quand je sors de la discussion avec la jeune fille, une journaliste du Parisien me poursuit en courant parce qu'elle veut faire son métier. Et elle me demande une petite interview parce qu'en fait apparemment elle n'a pas pu avoir d'interview des demoiselles sur place, qui n'avaient apparemment pas l'autorisation de parler à des médias directement...[...] Et je lui ai expliqué pourquoi j'avais pas peur. Finalement j'ai fait exactement la même chose avec ma maman et pourquoi ? Ben parce qu'ils ont pas forcément réfléchi... De toutes façons, eux, ils connaissent pas non plus dans le détail les choses. C'est pour ça, c'est logique, ils ont le droit d'avoir peur. Et quand on a les connaissances, on peut pas se permettre d'avoir peur, parce qu'on a les connaissances pour répondre à la question. »

«Quand on aura des résultats je leur en parlerai. Et puis je me dis que peut-être ça éveillera leur curiosité pour après, peut-être qu'un jour ils participeront à un essai clinique. »

\section{Dimension idéologique de l'ethos}

Les notions foucaldiennes de gouvernementalité et de biopolitique aident à aborder la question de l'idéologie dans le cas qui nous occupe. Rappelons que Foucault (2001 : 1547) distingue trois relations de pouvoir :

« Il me semble qu'il faut distinguer les relations de pouvoir comme jeux stratégiques entre des libertés - jeux stratégiques qui font que les uns essaient de déterminer la conduite des autres, à quoi les autres répondent en essayant de ne pas laisser déterminer leur conduite ou en essayant de déterminer en retour la conduite des autres -, les états de domination, qui sont ce qu'on appelle d'ordinaire le pouvoir.

Et, entre les deux, entre les jeux de pouvoir et les états de domination, vous avez les technologies gouvernementales, en donnant à ce terme un sens très large - c'est aussi bien la manière dont on gouverne sa femme, ses enfants que la manière dont on gouverne une institution. »

Et il ajoute :

«L'analyse de ces techniques est nécessaire, parce que c'est très souvent à travers ce genre de techniques que s'établissent et se maintiennent les états de domination. »

\subsection{L'engagement dans des essais vaccinaux comme technique de soi}

Ce qui intéresse les analystes du discours, c'est de saisir dans l'activité discursive du sujet les marques de la subjectivation à travers les traces de l'incorporation du " sens commun », c'est-à-dire l'expression des styles de vie, des pratiques imaginaires et des «techniques de soi » au sens où l'entend Foucault ${ }^{4}$. Les entretiens sont un lieu privilégié de réflexivité et, à ce titre, ils peuvent compter parmi les «techniques de soi » comme en témoignent les témoignages recueillis :

«Et surtout pour cette maladie là [...] pour les gens qui s'engagent là-dedans, d'un point de vue médical c'est l'œuvre de toute une vie quoi [...] m'impliquer

\footnotetext{
${ }^{4}$ Parmi les exemples de techniques de soi que Foucault évoque, on trouve - outre les hypomnemata (écriture de soi), la lecture, la méditation, la déambulation - les retraites, les régimes, les entraînements.
} 
personnellement quoi. Intimement on va dire. Parce que c'est le corps, c'est tout ça quoi. Parce que là, je vais le faire d'un point de vue professionnel, donc ça c'est une chose, mais après il y a l'intime aussi quoi. »

Il s'agit d'être attentif aux realia évoqués. Warnier (2004: 7), se réclamant d'une ethnographie de l'assujettissement que Foucault n'aurait pas développée, évoque la « culture matérielle incorporée » : elle conduit à s'intéresser aux choses elles-mêmes, car

«les choses matérielles très concrètes (...) s'inscrivent dans des processus de subjectivation qui, à tout le moins, constituent le socle des rapports politiques [...] l'analyse du politologue et de l'anthropologue doit prendre en compte le sujet, ses conduites sensorimotrices, ses inventaires de culture matérielle incorporée, ses goûts, dégoûts et passions. »

L'objectivation des corps que manifestent certains entretiens en est une des manifestations que nous appréhendons à travers trois séries d'indices :

- la désignation anatomique :

«Enfin, c'est pas banal non plus, on prête son corps quelque part. Moi je suis déjà un donneur de sang régulier aussi. »

Je pense que les cancers, tout ça, ça n'aurait pas du tout été la même chose. C'était vraiment le VIH [...]. J'ai donc j'ai été écartée du circuit. Ça m'a peiné parce que le sang, la moelle, tout ça...»

- l'évocation de l'astreinte qu'implique l'entrée dans le díspositif auquel les volontaires se soumettent librement. L'obligation de s'en tenir à des pratiques de vie saine exerce un fort impact sur la vie intime des individus (par exemple le respect de règles d'hygiène et de normes sexuelles).

- la mention de la nécessaire inscription dans la temporalité de l'expérimentation. Étant donné la planification sévère de l'emploi du temps, le dispositif médical régule leur quotidien. Les volontaires n'en aspirent pas moins à être retenus pour les essais et certains se déclarent prêts à planifier leur engagement à longue échéance.

«j'attendais d'avoir une visibilité plus grande - sur mon emploi du temps professionnel] - pour me lancer dans un truc qui, justement, me demandait de m'engager sur un an.»

Pour moi, même si pour l'instant je peux pas le faire, si jamais mon taux d'anémie remonte, puisque du coup je peux quand même aller voir un médecin pour faire remonter tout ça, j'envisage toujours de participer si ça remonte et qu'ils sont toujours en recherche. »

\subsection{Engagement politique informel de type pragmatiste et biopouvoir}

Les volontaires sont engagés à plus d'un titre : ils coopèrent à la recherche médicale à travers leur engagement physique et ils coopèrent à la politique de recrutement à travers leurs dires dans les entretiens. En retour, de telles actions permettent à l'individu de se construire et de maintenir une identité à travers les offres de sens du ministère de la santé et du chercheur (Glady 2008 : 55). Car comme tout sujet de langage, ils sont portés par des formations discursives dominées par l'interdiscours et comme l'écrit Bayart (2004:16) : 
«la subjectivité des acteurs, dont on s'accorde désormais à reconnaître l'importance, n'équivaut pas à celle de sujets autonomes et individuels, mais à un processus de subjectivation tel que l'entendait Foucault, en tant que processus de « constitution de sujets moraux » à travers la production de « styles de vie ». C'est moins le Sujet constitué qui compte que la constitution du Sujet ; moins l'action du Sujet que l'action qui fait le Sujet (et à laquelle participe l'action du Sujet. »

On peut se poser la question de savoir si l'on peut parler d'engagement militant dès lors que les locuteurs ne convoquent pas un collectif dont il seraient effectivement les membres car l'engagement ici a ceci de particulier qu'il est solitaire. Mais il est aussi solidaire : les paroles des volontaires renvoient à la conscience d'une participation à l'effort collectif, lequel repose sur un large consensus : ils sont convaincus de l'efficacité et du bien fondé de leur action et ils mettent en récit leur expérience en reprenant à leur compte la doxa sans exprimer de point de vue quant à la dimension idéologique de l'entreprise. Cependant, l'engagement dans les essais vaccinaux relève d'un type d'action "politique par le bas " (Bayart et al. 1992, Bayart 2008), c'est-à-dire d'une action qui n'est pas reconnue comme politique alors qu'elle implique pleinement le citoyen. Cette invisibilité s'apparente à de la « politique informelle », tel qu'il est défini par Le Gall, Offerlé et Ploux (2012:16) :

« Nous entendrons par « politique informelle » les formes, les pratiques, les expressions politiques qui, faute de bénéficier d'une reconnaissance et d'une légitimité de la part des prescripteurs et des agents du champ politique, sont " rejetées » en dehors de ce champ quand bien même elles participent, pleinement ou accessoirement, à sa constitution. À chaque époque sa «politique informelle » serions-nous alors tentés de dire dans la mesure où celle-ci se niche dans des espaces - sociaux, topographiques, discursifs [...] - qui adviennent en fonction des évolutions économiques et sociales et de leurs incidences sur l'ordre politique. »

Un tel engagement relèverait du « rapport pragmatiste à l'engagement » que Pereira (2007) distingue du « rapport total à l'engagement» et du « rapport esthétique individualiste » caractéristiques des modalités de militantisme dépassés aujourd'hui :

« Le caractère dominant de ce que nous avons appelé l'engagement pragmatiste aujourd'hui, nous semble constituer une tentative d'alternative à la fois au rapport total qui sacrifiait l'individualité au profit du collectif et au rapport esthète qui sacrifiait la solidarité et l'engagement collectif à l'affirmation de soi. L'engagement pragmatiste essaie de dépasser le dualisme entre une anthropologie communautaire et une anthropologie individualiste. Nous faisons donc l'hypothèse que l'engagement pragmatiste constitue une éthique. Il s'agit d'une éthique dans la mesure où l'engagement y serait vécu comme un moyen d'affirmation personnelle mais dans le cadre d'une anthropologie communautarienne. »

Certains des propos manifestent explicitement cette double motivation :

«Je le fais pas pour moi-enfin, on le fait toujours un peu pour soi, mais je le fais aussi surtout pour d'autres gens quoi. »

« [...] on a le droit de tous participer à l'essai clinique et tu peux être acteur de ce mouvement et t'investir pour une cause en fait. [...] Et être en fait acteur de sa santé, finalement. » 
Si avec le premier Foucault on peut considérer que ce choix relève de l'assujettissement, les acteurs ne faisant qu'investir des stéréotypes attachés à l'expérience qu'ils vivent et à un mode d'engagement socio-historiquement déterminé, cependant, comme l'affirment N. Elias et le Foucault dans L'Herméneutique du sujet, le bénéfice personnel de la construction de soi s'articule à la prise en charge de soi en vue de la bonne marche de la collectivité sur le mode de la délégation du biopouvoir à l'individu :

«Normalisation et autocontrôle individuel visent tous deux à obtenir une pacification interne de la société et une intériorisation des contraintes étatiques ou externes, qui s'achèvent en un retrait relatif de l'État sur ses fonctions régaliennes, au profit d'une prise en charge de nombre de ses fonctions par les individus. » (Taïeb 2005 : 40)

Le cadre contraint de l'entretien amène à questionner son appartenance à la collectivité et à investir les stéréotypes attachés à l'expérience. À travers l'expérience de l'expression de soi les volontaires se voient proposer une place et un statut comme acteur social. L'analyse de l'ethos discursif selon les dimensions expérientielle, catégorielle et idéologique aide à aborder de façon nuancée l'implication du profane dans l'expertise médicale et à cerner les spécificités d'une action solidaire/solitaire que l'on ne peut pas opposer radicalement à l'idée d' " engagement collectif» car elle illustre un nouveau type de rapport contemporain au politique. Cette étude prétend apporter sa contribution à un projet plus ambitieux concernant plus généralement les modes d'action politique informelle, sujet d'actualité en période de défection des partis politiques traditionnels et des structures syndicales.

\section{Bibliographie}

BAYART Jean-François, 2004, "Total subjectivation ", in BAYART Jean-François et WARNIER Jean-Pierre (dir.), Matière à politique. Le pouvoir, les corps et les choses, Paris, Karthala, 215-254.

BAYART Jean-François, 2008, « Comparer par le bas », Sociétés politiques comparées, 1-25. BAyART Jean-François, MBemBe Achille et Toulabor Comi M., 1992, Le Politique par le bas en Afrique noire. Contributions à une problématique de la démocratie, Paris, Karthala.

Charaudeau Patrick, 2005, «Sémantique de la langue, sémantique du discours », in Actes du colloque en hommage à Bernard Pottier, consulté le 4/12/2016, < http://www.patrickcharaudeau.com/Semantique-de-la-langue-semantique.html $>$

EBEL Marianne. 1981, "L'explication : acte du langage et légitimité du discours », Revue Européenne des Sciences Sociales, 56, 15-36.

FouCAUlt Michel, 1994, «Les techniques de soi » in Dits et écrits. 1954-1988, tome IV : 1980-1988, Paris, Gallimard, texte n 363.

FouCAUlT Michel, 2001, «L'éthique du souci de soi comme pratique de la liberté », Dits et écrits II. 1976-1988, Paris, Quarto-Gallimard, texte $\mathrm{n}^{\circ} 356$.

GLADY Marc, 2008, «Destination(s) de la connaissance dans l'entretien de recherche : l'inégale appropriation des offres de sens », Langage et société, 123), 53-72.

Le Gall Laurent, Offerlé Michel et Ploux François (dir.) (2012, La politique sans en avoir l'air. Aspects de la politique informelle, XIXe-XXIe siècle. Rennes, PUR.

MaIngueneau Dominique, 2004, "Hyperénonciateur et particitation », Langages, 156, 11 126.

MaIngueneau Dominique, 2014, " Retour critique sur l'éthos », Langage et société, 149, 31-48.

Maingueneau Dominique, 2013, «L'ethos : un articulateur », COnTEXTES [En ligne], 13, consulté le 04/12/2016, < http://contextes.revues.org/5772 ; DOI : 10.4000/contextes.5772> 
ORBIKI Eithan, 2015, «Le(s) discours de l'action collective : contextes, dynamiques et traditions de recherche », Argumentation et Analyse du Discours [En ligne], 14. Consulté le 03 décembre 2016, < http://aad.revues.org/2002>

PEREIRA Irène, 2007, «Individualité et rapports à l'engagement militant », ¿ Interrogations ?, 5. L'individualité, objet problématique des sciences humaines et sociales,

$<$ http://www.revue-interrogations.org/Individualite-et-rapports-a-l $>$

PINTO Louis, 2009, Le collectif et l'individuel. Considérations durkheimiennes, Paris, Raisons d'agir, coll. « Cours et travaux ».

TAÏ̈B Emmanuel, 2005, « Individuation et pouvoir politique », Labyrinthe, 22, 37-46.

WARNIER Jean-Pierre 2004, "Pour une praxéologie de la subjectivation politique », in BAYART Jean-François et WARNIER Jean-Pierre (dir.), Matière à politique. Le pouvoir, les corps et les choses, Paris, Karthala, 7-31.

\section{Résumé}

Cet article interroge le degré d'adhésion plus ou moins grand aux valeurs en vigueur dont témoignent la catégorie discursive de l'expression de soi. Si les dires de soi sont des lieux de réflexivité privilégiés, ils constituent également l'occasion de se positionner en fonction de normes propres aux champs dans lesquels ils évoluent : il s'agit de rendre visible et de légitimer un engagement, une position et/ou une existence. Dans le cadre d'entretiens semidirectifs menés auprès de volontaires engagés dans des essais vaccinaux, on amène les acteurs à verbaliser leur expérience, en vue d'améliorer les campagnés de recrutement dans le cadre d'une action de santé publique. Il s'avère que l'interaction témoigne du partage des valeurs et des convictions en faveur de l'engagement individuel pour la collectivité articulé au bénéfice personnel de la construction de soi.

\section{Abstract}

This article questions the degree of adherence to standard values that the discursive category of self-expression manifests. If self-discourses are privileged places of reflexivity, they also offer an opportunity to position oneself according to the standards of the fields in which they move : the point is to rich a certain degree of visibility for a commitment, a position or an existence to be legitimized (Honnett 2007; Heinich 2012; Delormas 2013). In order to improve recruitment campaigns within the framework of an action in the field of public health, clinical trial volunteers are interviewed (by semi-directed interviews) and encouraged to put their experience into words. It turns out that the volunteers make there motivation in a collective action known by generally seizing completely the researcher's offer of sense (Glady 2008: 55) : the interaction attests to shared values and convictions in favour of the individual commitment for the collectivity linked with self-construction representing a personal benefit. 